\title{
Water, juice, or soda? Mothers and grandmothers of preschoolers discuss the acceptability and accessibility of beverages
}

\section{Citation:}

Eli, K., Hörnell, A., Malek, M. E., \& Nowicka, P. (2017). Water, juice, or soda? Mothers and grandmothers of preschoolers discuss the acceptability and accessibility of beverages. Appetite, 112, 133-142.

\section{Publisher's version:}

http://dx.doi.org/10.1016/j.appet.2017.01.011

\begin{abstract}
Intake of sugary beverages is strongly associated with weight gain and obesity among children; however, differences between mothers' and grandmothers' attitudes and practices concerning young children's beverage consumption remain unclear. This is notable since about a quarter of families in the US and the UK rely on grandparents as the main providers of informal childcare. The aim of this study is to examine mothers' and maternal grandmothers' attitudes, knowledge, and practices regarding preschool aged children's beverage consumption. The analysis focuses on identifying intergenerational similarities and differences, given the potential impact that such differences might have on young children's beverage consumption habits. Twenty-two semi-structured interviews, representing eleven families, were analyzed using thematic analysis. The sample included all mother maternal grandmother dyads from The Grandparents Study, which took place in Eugene, Oregon, USA. More than half of mothers and grandmothers met overweight/obesity criteria. Among the children (mean age 4.7 years; five girls and six boys), seven met overweight/obesity criteria. Most mothers and grandmothers were unemployed, and most reported an annual household income below 30,000 USD. The analysis identified three thematic categories: 1) mothers and grandmothers agree about the hierarchy of healthiness between and within beverages, though juice occupies an ambivalent position; 2) mothers and grandmothers cite role modeling and the home environment as important in regulating preschoolers' beverage intake; 3 ) mothers and grandmothers balance between restricting sugary beverages and using these beverages as treats. The results suggest that when mothers and grandmothers use soda, juice, and juice-drinks as treats, they do so within a wider dynamic of balancing practices, and within two intersecting domains: the hierarchy of beverages, including the still ambivalent status of juice as healthy or unhealthy, and the definition of 'special occasion'.
\end{abstract}

\section{Keywords}

- Grandparents;

- Knowledge;

- Feeding practices;

- Sugar-sweetened beverages 


\section{Background}

Eating and drinking practices developed early in childhood may persist into adulthood (Nicklas, Yang, Baranowski, Zakeri, \& Berenson, 2003). Parents influence children's eating practices directly, through the foods they serve, and indirectly, through modeling eating behaviors and transmitting social norms about food and eating (Sleddens et al., 2011 and Larsen et al., 2015). Several models and frameworks have been developed to describe the complex processes of familial decision making about food - processes influenced by multi-level factors, including structural determinants. (Gillespie and Johnson-Askew, 2009, Lovell, 2016, Sobal and Bisogni, 2009 and Visser et al., 2016). Parental socioeconomic status (SES) is the most studied structural determinant of parents' and children's eating practices, and numerous studies have shown that children from households of lower SES are at higher risk for obesity (Shrewsbury \& Wardle, 2008) and that levels of parental education are associated with children's overweight or obesity in early childhood (Svensson et al., 2014 and Wijlaars et al., 2011). However, much less is known about how dynamics of care and communication between family members may influence healthy eating practices and their transmission within families of preschool aged children, including lower SES families (Blissett et al., 2011, Hurley et al., 2011, Larsen et al., 2015, Rollins et al., 2016 and Sleddens et al., 2011). It is also unclear how family members facilitate children's effective self-regulation practices through setting limits while avoiding excessive restriction (DiSantis et al., 2011 and Ventura and Birch, 2008). Self-regulation is important not only in eating practices (Rollins et al., 2016), but also with regard to the consumption of sugary beverages - a practice that continues to be very prevalent among children and that is linked to childhood obesity (Malik, Pan, Willett, \& Hu, 2013). Better insight into familial dynamics related to eating and drinking practices can inform the development of population-wide and personalized prevention and treatment programs for childhood obesity and related chronic conditions, such as type 2 diabetes.

One overlooked familial factor in young children's eating practices is the impact of grandparents. The grandparental role has been recognized as distinct since at least the early modern period (Bailey, 2012, Cressy, 1986 and Davis, 1977). While childhood obesity is a relatively recent public health concern, critiques of grandparental feeding are not new. In her monograph, Parenting in England 1760-1830: Emotion, Identity, and Generation, Bailey (2012) quotes a British physician who complained, in 1748:

it may serve to convince most Nurses, Aunts, Grand-mothers, \&c. how much they have hitherto been in the wrong, what Mischief is done to Children, and what Multitudes are destroyed or spoiled, as well by cramming them with Cakes, Sweetmeats, \&c. till they foul their Blood, choak their Vessels, pall the Appetite, and ruin every Faculty of their Bodies; as by cockering and indulging them, to the utter Perversion of their naturally good Temper, till they become quite forward and indocile. (Cadogan, 1748; cited in Bailey, 2012, p. 199)

Although concerns about grandparents' supposedly indulgent feeding might be implicit in public health and clinical interventions, grandparental feeding practices have only recently been subject to research (Eli et al., 2016 and Farrow, 2014). A systematic review found that parents cite grandparents as a barrier to maintaining healthy eating practices at home (Pocock, Trivedi, Wills, Bunn, \& Magnusson, 2009). Indeed, informal childcare by grandparents has been associated with higher proportions of overweight and obesity in children, while formal childcare seems to protect against childhood obesity (Benjamin et al., 2009, Kim and Peterson, 2008, Lumeng et al., 2005 and Maher et al., 2008); however, the processes underlying these differences have yet to be investigated. In approximately one quarter of US families (U.S. Census Bureau, 2010) and UK families (Rutter \& Stocker, 2014), parents rely primarily on grandparents for informal childcare. Thus, from a public health perspective, it is essential to examine grandparental attitudes and practices concerning child feeding. We therefore designed a qualitative study involving parents and grandparents in families with preschool aged children. 
We interviewed family members about their perceptions of and engagement with children's food habits, beverage habits, and physical activity, as well as their attitudes towards children's body weights. The present paper is the fourth in a series originating from the study, and the third to focus on the preschoolers (one paper focused on the adult family members' recollections of their own childhood body image [Eli, Howell, Fisher, \& Nowicka, 2014a]). The first of these papers studied potential generational differences between parents' and grandparents' perceptions of their preschoolers' body sizes (Eli, Howell, Fisher, \& Nowicka, 2014b). We found that neither parents nor grandparents tended to identify excess body weight in preschool aged children. Even when parents and grandparents did recognize that a preschooler was overweight, they rarely intervened. In the second paper, we set out to investigate the processes underlying parental and grandparental decisionmaking about preschoolers' feeding and physical activity (Eli et al., 2016). We found that, within each family, parents and grandparents described adjusting their feeding practices in relation to one another. Key to these adjustments were notions of balance. Feeding practices were informed by notions of "a balanced lifestyle" (Eli et al., 2016, p. 28), with participants citing healthy feeding as offsetting indulgent feeding. Moreover, "balanced lifestyle" practices were negotiated through familial homeostasis - the maintaining of balance between parents' and grandparents' care dynamics, with healthy and indulgent feeding practices used to enact differences between parental and grandparental caretaking roles (Eli, et al., 2016).

While parents' and grandparents' negotiations of preschool aged children's eating practices are grounded in notions of balance and care, it is unknown if the same notions apply to beverage-related practices. Beverages are often overlooked when lay people, and sometimes researchers, discuss children's food habits - perhaps because, although a variety of foods is needed in order to maintain nutritional balance, this is not the case with beverages. Once infants are weaned off breast milk (or breast milk replacements), the only beverage they require for continued thriving is water. Calorific beverages, then, provide children with energy that is not nutritionally required. In the literature, several terms are used to designate beverages that contain sugars (sucrose, glucose, or fructose), including 'sugary beverages' and 'sugar-sweetened beverages'. In the present study, we use the term 'sugary beverages' to refer to soda, juice drinks, $100 \%$ fruit juices, fruit and vegetable fusion juices, sports drinks, and sweetened flavored water; our chosen term reflects the fact that fruit juices - which contain considerable amounts of sugar - are not necessarily sugar-sweetened. However, we use the term 'sugar-sweetened beverages' when referring to articles that specifically employ this term or that concern the consumption of soda and other beverages with added sugars.

In recent years, the link between consumption of sugary beverages and body weight has become a matter of scientific and public interest, and decreasing children's consumption of sugary beverages is one of the main goals of public health initiatives world-wide (World Health Assembly Resolution WHA63.14, 2010). A systematic review and meta-analysis published in 2013 showed that consumption of sugar-sweetened beverages is associated with weight gain in children (Malik et al., 2013). The meta-analysis identified 20 studies concerning children's sugar-sweetened beverage consumption, and found that a single daily serving of sugar-sweetened beverages was associated with an increase of $0.06 \mathrm{~kg} / \mathrm{m}^{2}$ in body mass index (BMI) over the course of one year. Five of the 20 studies were randomized controlled trials; the studies found that, when sugar-sweetened beverage consumption was reduced, BMI was reduced by at least $-0.12 \mathrm{~kg} / \mathrm{m}^{2}$ (Malik et al., 2013). Notably, one experimental study used a double-blinded, randomized controlled trial design among normalweight children who were all habitual drinkers of sugar-sweetened beverages (de Ruyter, Olthof, Seidell, \& Katan, 2012). The children, aged 4-12 years, were randomized to receive either one serving of a sugar-sweetened beverage every day for 18 months or the same amount of an artificially sweetened beverage. The children in the sugar-sweetened beverage group had a greater increase in mean body weight compared to the children in the artificially sweetened beverage group $(7.37 \mathrm{~kg}$ in the sugar-sweetened beverage group vs $6.35 \mathrm{~kg}$ in the artificially sweetened beverage group). Another study showed that a high intake of sweetened beverages among 5-year-olds was linked with unhealthy 
eating habits later in life, including a "higher intake of added sugars, and lower intakes of protein, fiber, vitamin D, calcium, magnesium, phosphorous, and potassium" (Fiorito, Marini, Mitchell, Smiciklas-Wright, \& Birch, 2010, p. 547). Moreover, infants who consume sugar-sweetened beverages are more likely to consume such beverages at 6 years of age, suggesting that the consumption of sugar-sweetened beverages in early life may ingrain this practice as a pattern (Park, Pan, Sherry, \& Li, 2014).

Given the negative short-term and long-term consequences of consuming sugary beverages in excessive amounts, it is concerning that many children and adolescents habitually consume sugary beverages, including high-fructose corn syrup (HFCS) sweetened beverages, such that they substantially exceed the recommended daily limit of $25 \mathrm{~g}$ of sugar (World Health Organization, 2015). These beverages have proven to be the main source of added sugars among children and young people around the world (American Academy of Pediatrics, 2004, Danyliw et al., 2012 and Guthrie and Morton, 2000). The consumption of sugary beverages is high among preschool aged children (Nickelson, Lawrence, Parton, Knowlden, \& McDermott, 2014), and sugar-sweetened beverages represent one of the main sources of added sugars in children aged 2-5 years (Kranz, SmiciklasWright, Siega-Riz, \& Mitchell, 2005).

Parents have an important role in facilitating and regulating children's consumption of sugary beverages, but parental decision-making should be considered in environmental contexts. For example, a recent US-based study that examined perceptions of sugary beverages among parents of children and adolescents (ranging in age from 2 to 17 years old), found that most parents knew that sugary drinks are unhealthy (Munsell, Harris, Sarda, \& Schwartz, 2016). Despite this, Munsell et al. (2016) report that almost all participating parents gave their children sugar-sweetened drinks, having perceived fruit drinks, sports drinks, and flavored water as healthier alternatives to soda. The authors suggest that this misconception may be because the packaging and advertising of juice drinks, sports drinks, and flavored water makes these beverages appear healthier than they are. They also propose that this may reflect the inadequate advertising of public health recommendations to reduce children's sweetened beverage intake, and conclude that clearer public health messages are needed, to convey that these recommendations apply to all sugar-sweetened drinks, including juice drinks, and not just to drinks such as soda (Munsell et al., 2016).

The aim of the present study is to examine mothers' and maternal grandmothers' attitudes, knowledge, and practices regarding preschool aged children's beverage consumption, as expressed verbally. The study aims to identify intergenerational similarities and differences, and consider the potential impact that such differences might have on young children's beverage consumption habits.

\section{Methods}

This paper draws on data collected as part of "The Grandparents Study" (GP Study), which was conducted in 2011 at the Oregon Social Learning Center (OSLC), Eugene, Oregon, situated in the Pacific Northwest of the United States.

\subsection{Study population}

The total GP study population consisted of 49 family members (22 parents, 27 grandparents; $70 \%$ women, $30 \%$ men) from 16 families of children aged 3-5 years. Participants resided in Eugene, Oregon, a city with a population of approximately 142,000 inhabitants. The majority of children and adult participants in the GP study identified as white/Caucasian, reflecting the ethnic make-up of Eugene. Participants were recruited through advertisements published in a local newspaper and on Craigslist. The recruitment procedure has been explained in detail elsewhere (Eli et al., 2014a and Eli et al., 2014b). To be included, the participants had to be parents or grandparents of a child aged 3-5 years, who had no underlying medical condition or disability that affected her or his growth. Only families in which at least one parent and one grandparent agreed to be interviewed were included in 
the study. A primary inclusion criterion was the grandparent's active involvement in the child's life (defined as spending time with the child at least twice a month). Prior to the interview, each adult participant was asked to complete a sociodemographic questionnaire with detailed questions about family and living situations, education, and employment. Trained research staff weighed and measured all adult participants and their preschoolers.

The present paper draws on data collected through a convenience subsample of the GP Study's dataset, namely, all mother - maternal grandmother dyads $(n=11)$. These dyads were chosen because, in most families in the US (as well as in many European societies), mothers continue to play a dominant role in the provision of food and feeding (Blissett et al., 2006 and Khandpur et al., 2014), while maternal grandmothers have more contact with their grandchildren compared with (in decreasing order) maternal grandfathers, paternal grandmothers, and paternal grandfathers (Smith, 2005). Maternal grandmothers also occupy a privileged position in family caretaking dynamics, in the US and in many other societies (Smith, 2005). Therefore, an analysis that compares the beveragerelated discourses of maternal grandmothers with those of their daughters is particularly salient.

\subsection{Data collection}

Data were collected from February to July 2011 by the last author and by an undergraduate psychology student. The undergraduate student was employed as a research assistant and was trained in interview methods. The interview guide was developed to ensure similar techniques were used by both interviewers. Each of the adult participants took part in an individual, semi-structured interview lasting 1.5-2.5 hours. All participants were asked the same main questions, with adjustments to reflect the participants' parental or grandparental status. The interview guide included a list of possible follow-up prompts for each of the main questions, and follow-up questions were structured according to the participants' responses. The children were not present during the interviews; free child care was provided on-site when needed. All interviews were video recorded, in order to attend to body language. The video recordings of the interviews were also used to monitor interview quality throughout the data collection process. The interviews were transcribed verbatim by University of Oregon students. All parents and grandparents also answered a sociodemographic questionnaire concerning family structure, education, employment status, and income, among other variables.

The GP Study was approved by the Institutional Review Board of OSLC. The interviewers reviewed the consent forms with the participants when they arrived at OSLC, and answered any questions they had. The participants signed the project description and the project permission forms. All families received a copy of the informed consent forms and the written study description. Participants were informed that they could end their participation at any time without negative consequences and without explaining their reasons for leaving the study. They were also informed that all identifying data would be kept confidential in presentations and publications. Each interviewed participant received $\$ 50$ as compensation for participating in the study. The recruitment and interview procedures have been described in detail elsewhere (Eli et al., 2014a and Eli et al., 2014b). Codenames were used in all transcripts to ensure confidentiality.

\subsection{Analysis}

The analysis focuses on transcript excerpts that specifically concerned the consumption of beverages. Excerpts that concerned healthy eating, physical activity, and screen-time were excluded from the present analysis. The prompting question for the section of the interview guide that focused on beverages was: "So what does your child/grandchild like to drink?". This was followed by the questions: "And what does he/she drink most often?" and "What do you think about those choices? Good, bad? How come?". The participants were also asked: "Do you try to influence his/her choice of drink?". The excerpts were analyzed using thematic analysis (Braun \& Clarke, 2006). Two authors, $\mathrm{AH}$ and $\mathrm{KE}$, coded the texts independently; each of these two authors coded the texts in their entirety. 
Together with the last author (PN), they discussed the coded texts to identify patterns and cluster the codes into preliminary themes. Going back to the transcripts, the authors collated all representative text extracts under each preliminary theme. The authors also created a case-based table to note the appearance of the preliminary themes in each of the participant interviews, with each column representing a preliminary theme and each row representing a participant; the table was ordered by familial dyads, to identify potential intrafamilial convergence and divergence in preliminary themes. $\mathrm{AH}, \mathrm{KE}$, and PN discussed the preliminary themes again, until they reached consensus on combining these preliminary themes into the three thematic categories presented in the paper. The transcripts were reviewed again and comprehensive tables were created to collate all interview extracts relevant to each thematic category.

\section{Results}

The subsample included a total of 22 participants, representing eleven families (whose preschoolers in focus were five girls and six boys) (Table 1); the participants matched the total study population in both weight status and demographic characteristics. In the subsample, four children met BMI criteria for normal weight, two met BMI criteria for overweight, and five met BMI criteria for obesity, according to the US Centers for Disease Control and Prevention (CDC) 2000 growth charts (Krebs et al., 2007, Kuczmarski et al., 2000 and Kuczmarski et al., 2002). Two thirds of the mothers and more than half of the grandmothers met the WHO BMI criteria for overweight or obesity (World Health Organization, 2000).

Table 1.

Descriptive characteristics of the family members involved in the study.

$\begin{array}{llll} & \text { Children } & \text { Mothers } & \text { Grandmothers } \\ \text { Age (years) } & & & \\ & 4.7(3.1-5.7) & 31.3(22.7- & 55.11(44.8- \\ & & 40.2) & 64.1) \\ \text { Gender: Female/Male } & 5 / 6 & 11 / 0 & 11 / 0 \\ & 17.9(15.2- & 28.2(18.4- & 30.2(20.3-49.4) \\ \text { BMI }^{\mathbf{b}} & 21.5) & 39.1) & \end{array}$

\section{Weight status ${ }^{\text {b: }}$}

$\begin{array}{lccr}\text { Underweight } & 0 & 1 & 0 \\ \text { Normal weight } & 4 & 3 & 5 \\ \text { Overweight } & 2 & 3 & 2 \\ \text { Obese } & 5 & 4 & 4\end{array}$

\section{Ethnic background:}

$\begin{array}{llll}\text { White/Caucasian } & 9 & 11 & 9 \\ \text { Native American and white/Caucasian } & 1 & 0 & 2 \\ \text { Asian and white/Caucasian } & 1 & 0 & 0\end{array}$




\section{Education level:}

$\begin{array}{lccc}\text { High school only } & - & 1 & 0 \\ \text { Some college } & - & 7 & 9 \\ \begin{array}{l}\text { Standard 4-year college or university } \\ \text { graduation }\end{array} & - & 3 & 2 \\ \text { Employment status: } & - & 4 & 3 \\ \text { Full time } & - & 1 & 0 \\ \text { Part time } & - & 6 & 8 \\ \text { Not employed } & & & \end{array}$

a. Mean, min-max. All other numbers $=\mathrm{n}$.

b. For the children, weight status was determined according to the Centers for Disease Control and Prevention BMI criteria (overweight: 85th percentile $\leq$ BMI < 95th percentile; obesity: BMI $\geq 95$ th percentile) (Krebs et al., 2007, Kuczmarski et al., 2000 and Kuczmarski et al., 2002). For the adults, weight status was determined according to WHO BMI criteria (underweight: BMI <18.5; normal weight: BMI 18.5-24.9; overweight: BMI 25-29.9; obesity: BMI $\geq 30$ ) (World Health Organization, 2000).

The analysis resulted in three thematic categories: 1) mothers and grandmothers agree about the hierarchy of healthiness between and within beverages, though juice occupies an ambivalent position; 2) mothers and grandmothers cite role modeling and the home environment as important in regulating preschoolers' beverage intake; 3 ) mothers and grandmothers balance between restricting sugary beverages and using these beverages as treats.

Quotes from mothers and grandmothers that exemplify each of the thematic categories are provided in the text. Participant codenames indicate family number and the participant's relation to the child: for example, in Gp01P1 and Gp01G1, "Gp\#" stands for family number, "P" for "parent" (mother) and "G" for "grandparent" (maternal grandmother). The number after P and G reflects order of interview. "I" refers to the interviewer; "(...)" indicates that text had been removed for relevance and/or readability.

\subsection{Mothers and grandmothers agree about the hierarchy of healthiness between and within beverages, though juice occupies an ambivalent position}

The participants agreed on the hierarchy of healthiness between and within beverages. The major concern mentioned by participants was sugar content; fat content (in milk) was mentioned less frequently. Soda was the beverage cited most frequently as unhealthy. Water was the beverage cited most frequently as healthy; with the exception of one grandmother (Gp01G1), all participants mentioned water as a healthy beverage. When participants said their preschoolers liked water, they described this in positive terms:

I really love the fact that she loves water. We introduced that to her young so that's probably why. (Gp09P1)

Soda, on the other hand, was always described as unhealthy. It was rarely allowed, and then only as a 'treat' and in small amounts: 
I think if he had his choice, he would probably pick ... the forbidden thing is soda, so every once in a while his grandparents will let him have soda, so that would probably be his ... I guess it would be his favorite. (Gp03P1)

While most mothers and grandmothers also said milk was a healthy beverage and rich in nutrients, several participants said that low fat milk was preferable, and a few perceived cow's milk as unhealthy.

Participants expressed some misconceptions about juice, with discrepancies sometimes reflecting a generational divide. Some participants, particularly grandmothers, viewed juice as healthier than soda; in a few cases, this extended to lemonade and smoothies as well:

[Participant describing a restaurant situation] And then they'll pick like, a lemonade to drink instead of pop, which I think is wonderful! (Gp01G1)

Some of the grandmothers said that society-wide nutrition knowledge had improved over the years, and that nutrition knowledge was more accessible than it had been when they were the mothers of young children. They described how, as grandmothers, they had changed their beverage-related attitudes and practices:

When [daughter] was younger, Ijust thought, "Oh, juice is good, let her drink as much as she wants." (...) I used to have a picture of her, carrying this gallon jug apple juice with her, with her bottle. 'Cause we thought that was OK. Now we try to ... not let them drink as much juice ... (Gp05G1).

Some mothers described how education (either through their own college/university studies, or through The Special Supplemental Nutrition Program for Women, Infants and Children [WIC] groups) had changed their view of the healthiness of juice from an unreservedly positive to a more qualified view. Most mothers said that juice should be diluted or given in restricted amounts. Some grandmothers said they qualified their views of juice following discussions with their daughters. For example, one grandmother said:

We've learned that too much juice is just as bad in a way, as too much sugar or soda. (Gp05G1)

However, perceptions of mixed fruit and vegetable juices - namely, V8-Fusion - were mainly positive, despite their high fructose content. One mother and three grandmothers specifically mentioned V8-fusion as an easy way to ensure that the children reached their daily requirement of fruit and vegetable intake:

So if your kids aren't getting their fruits and vegetables they get a serving with each eightounce drink. So we make sure ... we've been buying that in different flavors and stuff so she gets that for lunch. So if we can't get the fruits and vegetables in her we know she is still getting the nutrients and stuff that she needs. (Gp01G1)

Mothers and grandmothers tended to agree on the kinds of beverages their child/grandchild preferred, most commonly soda, juice, water, and milk. While soda was mentioned as a favorite, the participants indicated that it was rarely allowed:

\section{He of course likes pop, but I don't give him pop. (Gp12P1)}

Juice was also among the children's preferred drinks, but most participants said they restricted juice consumption. Unlike soda, however, juice was allowed, usually in measured or diluted amounts, with concerns about sugar content moderated by the apparent 'healthiness' of $100 \%$ fruit juices. One grandmother described diluting juice with seltzer water [carbonated water] to make a healthier imitation 'soda'. A few participants cited only juice drinks that contained added HFCS as 
problematic, in contrast to 'natural' fruit juices and V8-fusion. One grandmother stated that her granddaughter could drink as much juice as she wanted as long as it did not contain HFCS:

She likes orange juice, apple juice, any, I mean she loves juice. She drinks that a lot. I: And can she drink as much juice as she wants? G: Yes, because we make sure there's no highfructose corn syrup in it. (Gp07G1)

Although nearly all mothers and grandmothers described their child/grandchild as liking sugary drinks, they did not describe struggles to make children drink water or milk. In fact, most participants mentioned water among their preschoolers' favorite drinks.

\subsection{Mothers and grandmothers cite role modeling and the home environment as important in regulating preschoolers' beverage intake}

Participants described various strategies to moderate or restrict their preschoolers' intake of sugary beverages. Generally, mothers expressed greater concern and spoke about setting 'rules' for beverage consumption, while grandmothers expressed less concern but tended to follow their daughters' lead. Some participants described their preschoolers as inclined toward sugary beverage consumption and thus needing more restriction and control, while others did not. All mothers and most grandmothers used a combination of strategies to encourage or discourage the consumption of particular beverages, which included varying degrees of overt and covert practices. Many preferred taking actions that the child would not notice, such as diluting juice or using skim or low-fat milk. Some mothers and grandmothers said that prohibiting a beverage entirely could be counterproductive:

I usually just say, "That's a sometimes food." It's not like I never ever let him have things, because I feel like if you have forbidden things, that's what they are going to focus on. (Gp04P1).

Participants highlighted the importance of modeling healthy beverage choices to their preschoolers. Some mothers described generational differences in role modeling, citing episodes from their own childhoods to contrast their parents' approach with their own. One mother, who spoke of modeling water consumption, said:

I think that she should drink more water but I try to encourage that like I'll drink water or I'll carry a water bottle with us when we go out (...). So that helps to promote that I drink water because my mom was always, "You drink water!" but she's drinking a soda ... so I'm like, that's not very fair so I try to promote that with my kid that I drink water too. (Gp11P1).

She continued, saying that she avoided drinking soda at home and that she restricted her soda consumption to a space her child did not access - her university campus. The maternal grandmother of the same child described how she had changed her attitude towards role modeling after witnessing her daughter's strategies; she added that she now tried to be a better role model for her grandchildren.

Many participants said they controlled the children's intake of sugary beverages by avoiding having these beverages in the house, and by making sure that healthy drinks were available and accessible:

The most effective thing to do is have the proper choices available, what's in the refrigerator, what's at hand for them to eat. Make sure that's healthy stuff; don't have soda and chips, because that's what they're going to go for. (Gp07G1)

Several mothers and grandmothers described how they made sure that their preschoolers had easy access to healthy choices, and pointed at the importance of making water the default choice. For example, one mother described the easy availability of water on kitchen counters as key to ensuring her children's healthy beverage consumption: 
I always make sure they have their water bottles on the counter where they can get them. (Gp14P1).

In a separate interview, her mother offered a contrasting account of how the easy availability of juice and soda at home can be detrimental to a child's health:

I think that sometimes kids will not drink water, because all they ever get is juice. (...) My nephew got seriously dehydrated because he wouldn't drink water, because there was pop and juice around. (...) I: Do you try to influence [grandchild]'s choice of drink at all? G: Only in what we have available. (Gp14G1).

In another case, a mother described encouraging her daughter to use a refrigerator water lever, as this technology made her child associate drinking water with a sense of independence:

I have a big thing in the fridge that she can pull the lever and get water out of so I try to promote it because she thinks it's cool that she can get her own water so we try to promote that. (Gp11P1)

Most mothers and grandmothers highlighted the importance of consistent rules and communication about healthy eating and drinking outside the home, and teaching children about nutrition so that they would be equipped to make the right choices themselves:

Just by explaining to him the differences in children's bodies and adult bodies. You know that his brain and his muscles are growing right now and if there is too much sugar or caffeine that it interferes with his growing. (Gp06G1)

Some mothers and grandmothers pointed out that the home was only one part of a child's food environment, and that spaces outside the home - specifically, schools - increasingly influence children's beverage consumption over time. These participants called for interventions and actions from schools and the government to facilitate healthy beverage choices:

You have to get the government to help you because no matter what you do, the kids, even if you start early, as the kids grow up there's peer pressure and you go to high schools and there's soda machines and there's junk food machines and what's going to happen? (Gp01G1)

Some participants also suggested that lowering the prices of healthy foods and beverages would be a way for the government to intervene and affect beverage consumption positively.

\subsection{Mothers and grandmothers balance between restricting sugary beverages and using these beverages as treats}

All mothers and the majority of grandmothers restricted soda and similar sugary beverages (e.g., Capri-Sun and Kool-Aid), which they perceived as unhealthy. These restricted drinks were also cited by most participants as 'treats' or 'rewards'. However, while soda was clearly mentioned as both a beverage to be restricted and as a treat, other sugary beverages - in particular, juice - were subject to a more complex depiction. Indeed, the hierarchy of beverages (described in Theme 1) influenced which drinks were seen as treats. Some participants spoke of juice (namely, V8-Fusion and 100\% juice) as part of the child's regular consumption of 'healthy' beverages; in these participants' discussions, juice was not depicted as a treat. However, other participants spoke of juice as a treat and specifically, a 'healthier' treat compared to soda. This ambivalent categorization of juice influenced the ways in which restriction and reward materialized in the mothers' and grandmothers' discussions.

Not all participants spoke of rewarding children with sugary drinks; however, those participants who said they provided sugary drinks as 'treats' usually linked these drinks with 'special occasions'. Notably, the definition of special occasions varied between families and individuals, and even within 
individual interviews. For example, one mother spoke about Capri-Sun as a beverage given on special occasions, but as her account progressed, it became apparent that Capri-Sun was provided daily as an after-school treat:

I: So what does she like to drink? M: Juice. I've never let her have soda (...). I: So do you try to limit juice? M: Yeah (...) her special thing is we buy juice and then every month when we go shopping I buy her a box or two of Capri-Suns and those are special drinks like maybe not even once a day like, "Do you want this kind of juice?" "Oooo ... special." Like usually when we get home from school she gets one. (Gpl1P1)

Along similar lines, the child's grandmother (GP11G1) said the child was given soda only on "special, special occasions", connoting a subtle qualitative difference between special occasions; however, she did not define what these occasions were.

Although some mother - grandmother dyads expressed similar views on restricting sugary beverages, in other cases, while one member of the dyad spoke only of restricting sugary beverages, the other spoke of using sugary beverages as treats or rewards. In some families, this disagreement followed the expected division between 'restricting' mothers and 'indulgent' grandmothers. Most grandmothers deferred to the mothers' decisions about what beverages to give the children. Some referred, however, to grandparents' prerogative to indulge grandchildren through providing them with 'treats' such as juice or soda when they spent time with them. Some mothers ignored their mothers' 'indulgent' beverage provision, especially when children spent a relatively small amount of time with their grandparents. For example, in family seven, the mother and grandmother differed in their attitudes to juice, with the mother deciding to restrict the child's juice intake, and the grandmother continuing to provide juice and juice-drinks as treats. Describing her past juice provision practices, the mother said:

You know, when she was younger, before I found out some things about nutrition, I used to give her a lot of juice, she would probably drink three or four of those six ounce boxes of juice a day. Which was way too much (...). (Gp07P1).

This mother now limited her daughter's juice consumption to $6-8$ oz per day. Yet her mother, who looked after the child on a daily basis, placed no restriction on juice as long as it did not contain HFCS - a rule she sometimes set aside:

Well she goes for the bad juice, I always read the bottle, high-fructose corn syrup, now I encourage her to get something else. If she's really insistent on a certain thing, I'll get it for her. I don't figure it's going to hurt her too bad; we try and throw the bottle away before [daughter] gets home (laughs). If she finds it she gives me the look, and then we're ok. I claim grandmother privileges. (Gp07G1)

Key to this grandmother's explanation was the phrase "grandmother privileges", through which she recast "bad" HFCS-sweetened juice as a treat. Yet, in some families, it was the grandmother who expressed stricter views about beverage provision, while the mother described providing sugary beverages as treats. For example, in one family, the maternal grandmother talked about providing her grandchild with low fat milk and did not mention using beverages as treats, while the mother described how the child's father, who saw him once a week, provided him with sugary drinks when they spent time together, and mentioned that she herself gave the child sugary beverages as a treat after dinner:

He likes to drink milk, he does like water, and he likes Blue Juice [Authors' note: probably a reference to a sports drink]. (...) Milk, he has to have milk at dinnertime. Blue Juice is on occasion, he has to have his milk then he can have his Blue Juice. (GP12P1) 
In another family (Gp13), the grandmother said she provided the child with V8 juice for the vitamins, and used Ovaltine as a substitute for ordinary chocolate milk to decrease fat and sugar intake.

However, the mother said she took the child to a fast-food restaurant, where he consumed soda, about once a week:

\section{He'll get upset if you don't take him out to eat. (...) He wants the toy and the drink, but he doesn't necessarily want the food. (Gp13P1)}

Notably, while this mother did not frame going to a fast-food restaurant as a special occasion, and instead cast it as a way of placating the child, she still framed soda as a treat equivalent to a toy, with food (in this case, processed meat) playing only a tangential role.

\section{Discussion}

Our study found that mothers and grandmothers are aware of the problematic status of sugary beverages, although some still perceive juice as healthy. Food and beverage practices, however, are multifaceted phenomena, which do not depend solely on perceived healthiness or unhealthiness. As the results show, mothers and grandmothers contextualize the provision of different types of beverages through notions of time and balance. Thus, while mothers and grandmothers may identify a sugary beverage as unhealthy, they may also identify the occasional provision of this beverage as a 'treat' to mark a 'special occasion' as an acceptable practice of care.

One of the key findings in this study was that all mothers and grandmothers combined several strategies to influence the children's intake of beverages, including role modeling, limit setting, and promoting independence. The strategies described by the participants were aligned with the literature on effective feeding practices (Rollins et al., 2016). Participants emphasized strategies of moderate control, wherein they regulated their preschoolers' intake of sugary beverages through supervisory practices - for example, by setting daily limits for juice and tracking the children's intake. Previous research has found that supervisory feeding practices are associated with children's lower intake of sugar-sweetened beverages (van der Horst et al., 2007). Participants also described combining limit setting with child-responsive practices - for example, setting a limit on sugary beverages while making water easily accessible within the home, thus increasing the children's sense of independence and reinforcing water as their beverage of choice in response to thirst cues. Earlier studies have found that such strategies, characterized in the literature as 'authoritative parenting', lead to lower BMI, more physical activity, and healthier eating among young children (Sleddens et al., 2011).

Of particular note in the present study was the ambivalent status of juice. While many participants particularly mothers - cited juice as unhealthy, some cited it as healthy or as healthier than soda. We therefore suggest that juice occupied a liminal position - "betwixt and between" categories ( Turner, 1967, p. 93) - and could not be subsumed into one defined beverage category. Indeed, juice was categorized alternately as a special 'treat', a beverage to be consumed regularly, and a healthy option in comparison to sugar-sweetened beverages. In line with Lovell's framework (Lovell, 2016), which builds on the models by Sobal and Bisogni (2009) and Gillespie and Johnson-Askew (2009), the present study highlights the importance of understanding personal and familial definitions of 'health' in obesity prevention and intervention efforts. The liminality, or 'in-between' status, of juice reflected the mixed messages to which participants were exposed. The great majority of mothers expressed concerns about the sugar content of juice, and some said they learned about child nutrition through the structured settings of college or WIC classes; however, $100 \%$ fruit juice is included in the WIC food lists. Likewise, while most participants were aware of the problematic sugar content of juice, several cited V8-Fusion as a good replacement for children's '5-a-day' fruit and vegetable consumption, likely reflecting the effect of successful marketing on the part of Campbell's (which has been accused of "deceptive" advertising of V8 products in 2014 ( Center for Science in the Public Interest, 2014)). Indeed, Munsell et al. (2016) found that food packaging and advertising texts lead to misconceptions 
among parents about the healthiness of beverages. The participants' views of juice, then, did not simply reflect knowledge or lack thereof, but were embedded in a broader structural framing of juice that involves both government initiatives and corporate marketing.

When mothers and grandmothers framed juice and other beverages as 'treats', notions of balance entered into their decision-making about providing sugary drinks. As we found in a previous study (Eli et al., 2016), for the parents and grandparents of preschoolers, establishing balance between foods and between caretaking roles was a key element of food provision. In the present study, participants spoke of providing soda, juice-drinks, and (in some cases) juice as part of 'special occasions', balancing out the occasional provision of 'unhealthy' beverages with the regular provision of beverages they labelled healthy (such as water and milk). Indeed, several mothers and grandmothers said that forbidding some beverages completely would be counterproductive as it would induce interest in these beverages, while also making it difficult for children to learn how to set limits on their own. Similar views were reported by Gram \& Grønhøj (2015), whose study of Danish parents' and children's reasoning about candy found that parents viewed the strict provision of healthy foods as potentially leading their children to unhealthy diets. Moreover, in the present study, mothers and grandmothers provided sugary beverages within the wider dynamic of caretaking roles. Some grandmothers spoke of 'spoiling' grandchildren with sugary beverages (as well as non-sugary beverages, such as herbal tea), as part of marking time spent together, thereby enacting their expected grandparental role as liminal caretakers (Farrow, 2014). In other families, grandmothers spoke of restricting children's sugary beverage consumption, or providing children with beverages they perceived as nutritious (such as V8-Fusion), implicating greater involvement in day-to-day caretaking. In a few cases, the mothers themselves spoke of providing children with sugary beverages as treats, while the grandmothers did not. These results suggest that balancing dynamics may be as applicable to beverages as they are to foods, and that the provision of sugary beverages in families of preschoolers should be examined within wider familial contexts (including fathers and grandfathers) to discern further how beverages are used in enacting caretaking roles. Notably, while previous research has found that parents often reference other parents' child feeding practices, rather than dietary guidelines, when describing their own feeding practices ( Duncanson, Burrows, Holman, \& Collins, 2013), in the present study, while mothers and grandmothers often referred to one another's beverage provision practices when describing their own, they did not refer to the child feeding practices of others outside the family. A number of participants, moreover, directly referred to nutrition classes as having informed their beverage provision choices, suggesting that participants tended to establish either 'authoritative' or familial reference points for their own practices.

\section{Strengths and limitations}

As with any other interview-based study, this study's conclusions are limited by the self-reported character of the data. Previous research has found that mothers' reports on controlling feeding and independent observations of the use of controlling feeding practices do not always match (Farrow, Blissett, \& Haycraft, 2011). However, the present study did not aim to establish an 'objective' truth of mothers' and grandmothers' practices, but rather to examine how they negotiate beverage-related attitudes and practices in discourse. One limitation was the study's focus on mothers and maternal grandmothers. While all mothers and maternal grandmothers played central caretaking roles, further research should include other adult caretakers, as the generalizability of the results to fathers and grandfathers is limited. Another limitation to generalizability is posed by the sample's lack of ethnic diversity, as the majority of participants identified as white/Caucasian, representing the ethnic distribution of Eugene, Oregon. The sample also had limited educational diversity, with most participants having completed high school education only. Future work should strive for a more educationally diverse sample, as low levels of education have been linked to excess adiposity in adults and children (McLaren, 2007 and Shrewsbury and Wardle, 2008), and higher levels of education have been linked to nutrition knowledge (Norman, Berlin, Sundblom, Elinder, \& Nyberg, 2015). Finally, 
the study's Oregon location was likely reflected in the discussions, or lack thereof, concerning cow's milk. While cow's milk was seemingly negligible to many participants, this finding should not be generalized beyond the Pacific Northwest, and we anticipate that in other US states and outside the US, cow's milk would have been mentioned more frequently. For example, a systematic review of the literature on the protein intake of children in the Nordic context has shown that, in Nordic countries, children consume cow's milk in large amounts, with cow's milk usually seen as a nutritional necessity for young children (Hörnell, Lagstrom, Lande, \& Thorsdottir, 2013). Excessive intake of cow's milk during the first two years of life could increase the risk for overweight and obesity in childhood and adolescence (Hörnell et al., 2013), and it is therefore important to conduct studies in milk-oriented societies, to explore how parents and grandparents construe cow's milk and the provision thereof.

\section{Conclusion}

While mothers and grandmothers generally agreed about the hierarchy of healthiness between and within beverages, juice continued to occupy an ambivalent position, and was framed variably as 'unhealthy', 'healthy', or 'healthier than soda'. This variability in the framings of juice reflected the intersections of policy and food corporations, which together propagate mixed messages about juice. Overall, when mothers and grandmothers perceived certain beverages as unhealthy, they spoke of these beverages as restricted - either entirely, or to be provided as 'treats' on 'special occasions'. Notions of balance emerged in participants' discourses of 'regular' versus 'special' beverage consumption, as well as in some families, where mothers and maternal grandmothers spoke of diverging beverage provision practices. The results suggest that when mothers and grandmothers use soda, juice, and juice-drinks as treats, they do so within a wider dynamic of balancing practices, and within two intersecting domains: the hierarchy of beverages, including the still ambivalent status of juice as healthy or unhealthy, and the definition of 'special occasions'.

Notably, our study suggests that nutrition knowledge may intersect with notions of balance and structural factors, such as economic insecurity and lack of subsidized/affordable childcare. While public health and clinical interventions frame food choices as essentially rational, reflecting knowledge or lack thereof - as highlighted by Lovell (2016) and Visser et al. (2016) - our study suggests that knowledge is only one component of decision-making about sugary beverages. Effective policy changes should take into account macro-scale factors - such as the advertising of juice as healthy and the inclusion of juice in WIC food lists, which could be subject to further regulation. Clinical and public health practitioners should acknowledge the role of caretaking dynamics within families, and the economic necessities that underlie reliance on grandparents for childcare (such as parents' underemployment, salaries below the living wage, and financial insecurity), leading parents and grandparents to engage in a sometimes-tense balancing of roles, enacted through variable provision of sugary beverages. Clinical interventions should also include critical media literacy training for adult caretakers, as well as practical strategies, such as how to moderate the serving of juice and other sugary drinks that may be perceived as healthy, and how to regulate the occasions when a child is served a sugary drink as a treat. Further research is needed to establish what constitutes a 'special occasion' which parents and grandparents would identify as cause for a 'treat', and how such concepts could best be addressed in public health and clinical practice. 


\section{Authors' contributions}

KE coded the interviews and analyzed them together with $\mathrm{AH}$, wrote and revised the manuscript, and approved the final manuscript as submitted. AH coded the interviews and analyzed them together with $\mathrm{KE}$, made a substantial contribution to the study's conception and design and to the writing of the manuscript, and approved the final manuscript as submitted. MEM contributed to the analysis and to the writing of the manuscript, and approved the final manuscript as submitted. PN conceptualized and designed the study, coordinated and supervised data collection and analysis, contributed to the writing and revising of the manuscript, and approved the final manuscript as submitted.

\section{Acknowledgements}

We thank all the parents and grandparents who took part in the Grandparents Study. We also thank Eliah Prichard, Jessica Farmer, Kelly Underwood, Bryn Shepherd, and Waihan Leung, the University of Oregon students who transcribed the interviews. We are grateful to Phil A. Fisher and Kyndal Howell, who both contributed to the conception and design of the Grandparents Study. Additional thanks go out to Joanne Bailey for her helpful input regarding historical perspectives on grandparenting. The study was supported by grants to PN from the Sweden-America Foundation, the Oregon Social Learning Center and the Marie Curie VINNMER International Qualification (201103443), and by a grant to MEM from the Department of Clinical Nutrition, Karolinska University Hospital. 


\section{References}

American Academy of Pediatrics (2004). "Policy statement: Soft drinks in schools." Pediatrics 113(1): 152-154.

Bailey, J. (2012). Parenting in England 1760-1830: Emotion, identity, and generation. Oxford University Press, Oxford.

Benjamin, S. E., S. L. Rifas-Shiman, E. M. Taveras, J. Haines, J. Finkelstein, K. Kleinman and M. W. Gillman (2009). "Early child care and adiposity at ages 1 and 3 years." Pediatrics 124(2): 555-562.

Blissett, J., C. Meyer and E. Haycraft (2006). Maternal and paternal controlling feeding practices with male and female children. Appetite 47(2): 212-219.

Blissett J, Meyer C, and E. Haycraft (2011). The role of parenting in the relationship between childhood eating problems and broader behaviour problems. Child Care Health Dev 37(5):642-8.

Braun, V. and V. Clarke (2006). "Using thematic analysis in psychology." Qualitative Research in Psychology 3(2): 77-101.

Center for Science in the Public Interest (2014). Campbell facing lawsuit over deceptive V8 juice drink marketing. https://cspinet.org/new/201406121.html, Center for Science in the Public Interest.

Cressy, D. (1986). Kinship and kin interaction in early modern England. Past \& Present, (113), 38-69.

Danyliw, A. D., H. Vatanparast, N. Nikpartow and S. J. Whiting (2012). "Beverage patterns among Canadian children and relationship to overweight and obesity." Appl Physiol Nutr Metab 37(5): 900906.

Davis, N. Z. (1977). Ghosts, kin, and progeny: Some features of family life in early modern France. Daedalus, 87-114.

de Ruyter, J. C., M. R. Olthof, J. C. Seidell and M. B. Katan (2012). "A trial of sugar-free or sugarsweetened beverages and body weight in children." N Engl J Med 367(15): 1397-1406.

Ek, A., K. Sorjonen, J. Nyman, C. Marcus and P. Nowicka (2015). "Child behaviors associated with childhood obesity and parents' self-efficacy to handle them: confirmatory factor analysis of the Lifestyle Behavior Checklist." Int J Behav Nutr Phys Act 12: 36.

Eli, K., K. Howell, P. A. Fisher and P. Nowicka (2014). "'A little on the heavy side": a qualitative analysis of parents' and grandparents' perceptions of preschoolers' body weights." BMJ Open 4(12): e006609.

Eli, K., K. Howell, P. A. Fisher and P. Nowicka (2014). ""Those comments last forever": parents and grandparents of preschoolers recount how they became aware of their own body weights as children." PLoS One 9(11): e111974.

Eli, K., K. Howell, P. A. Fisher and P. Nowicka (2016). "A question of balance: Explaining differences between parental and grandparental perspectives on preschoolers' feeding and physical activity." Social Science \& Medicine 154:28-35.

Farrow, C. (2014). "A comparison between the feeding practices of parents and grandparents." Eat Behav 15(3): 339-342. 
Farrow, C., J. Blissett and E. Haycraft (2011). Does child weight influence how mothers report their feeding practices? Int J Pediatr Obes 6(3-4): 306-313.

Fiorito, L. M., M. Marini, D. C. Mitchell, H. Smiciklas-Wright and L. L. Birch (2010). Girls' early sweetened carbonated beverage intake predicts different patterns of beverage and nutrient intake across childhood and adolescence. J Am Diet Assoc 110(4): 543-550.

Gerards, S. M., E. F. Sleddens, P. C. Dagnelie, N. K. de Vries and S. P. Kremers (2011). "Interventions addressing general parenting to prevent or treat childhood obesity." Int J Pediatr Obes 6(2-2): e28-45.

Gillespie, A. M. and W. L. Johnson-Askew (2009). "Changing family food and eating practices: the family food decision-making system." Ann Behav Med 38 Suppl 1: S31-36.

Gram, M. and A. Gronhoj (2015). "“There is usually just one Friday a week"." Food, Culture \& Society 18(4): 547-567.

Guthrie, J. F. and J. F. Morton (2000). "Food sources of added sweeteners in the diets of Americans." J Am Diet Assoc 100(1): 43-51, quiz 49-50.

Hörnell, A., H. Lagstrom, B. Lande and I. Thorsdottir (2013). "Protein intake from 0 to 18 years of age and its relation to health: a systematic literature review for the 5th Nordic Nutrition Recommendations." Food Nutr Res 57.

Hurley, K. M., Cross, M. B. and S. O. Hughes. (2011). A systematic review of responsive feeding and child obesity in high-income countries. J Nutr 141(3): 495-501.

Khandpur, N., R. E. Blaine, J. O. Fisher and K. K. Davison (2014). "Fathers' child feeding practices: a review of the evidence." Appetite 78: 110-121.

Kim, J. and K. E. Peterson (2008). "Association of infant child care with infant feeding practices and weight gain among US infants." Arch Pediatr Adolesc Med 162(7): 627-633.

Kranz, S., H. Smiciklas-Wright, A. M. Siega-Riz and D. Mitchell (2005). "Adverse effect of high added sugar consumption on dietary intake in American preschoolers." J Pediatr 146(1): 105-111.

Krebs, N. F., J. H. Himes, D. Jacobson, T. A. Nicklas, P. Guilday and D. Styne (2007). Assessment of child and adolescent overweight and obesity. Pediatrics 120 Suppl 4: S193-228.

Kuczmarski, R. J., C. L. Ogden, L. M. Grummer-Strawn, K. M. Flegal, S. S. Guo, R. Wei, Z. Mei, L. R. Curtin, A. F. Roche and C. L. Johnson (2000). CDC growth charts: United States. Adv Data(314): $1-27$.

Kuczmarski, R. J., C. L. Ogden, S. S. Guo, L. M. Grummer-Strawn, K. M. Flegal, Z. Mei, R. Wei, L. R. Curtin, A. F. Roche and C. L. Johnson (2002). 2000 CDC Growth Charts for the United States: methods and development. Vital Health Stat 11(246): 1-190.

Larsen, J. K., R. C. Hermans, E. F. Sleddens, R. C. Engels, J. O. Fisher and S. P. Kremers (2015). How parental dietary behavior and food parenting practices affect children's dietary behavior. Interacting sources of influence? Appetite 89: 246-257.

Lovell, J. L. (2016). How parents process child health and nutrition information: A grounded theory model. Appetite 97: 138-145. 
Lumeng, J. C., K. Gannon, D. Appugliese, H. J. Cabral and B. Zuckerman (2005). Preschool child care and risk of overweight in 6- to 12-year-old children. Int J Obes (Lond) 29(1): 60-66.

Maher, E. J., G. Li, L. Carter and D. B. Johnson (2008). Preschool child care participation and obesity at the start of kindergarten. Pediatrics 122(2): 322-330.

Malik, V. S., A. Pan, W. C. Willett and F. B. Hu (2013). Sugar-sweetened beverages and weight gain in children and adults: a systematic review and meta-analysis. Am J Clin Nutr 98(4): 1084-1102.

McLaren, L. (2007). Socioeconomic status and obesity. Epidemiol Rev 29: 29-48.

Munsell, C. R., J. L. Harris, V. Sarda and M. B. Schwartz (2016). Parents' beliefs about the healthfulness of sugary drink options: opportunities to address misperceptions. Public Health Nutr 19(1): $46-54$.

Nickelson, J., J. C. Lawrence, J. M. Parton, A. P. Knowlden and R. J. McDermott (2014). What proportion of preschool-aged children consume sweetened beverages? J Sch Health 84(3): 185-194.

Nicklas, T. A., S. J. Yang, T. Baranowski, I. Zakeri and G. Berenson (2003). Eating patterns and obesity in children. The Bogalusa Heart Study. Am J Prev Med 25(1): 9-16.

Norman, A., A. Berlin, E. Sundblom, L. S. Elinder and G. Nyberg (2015). Stuck in a vicious circle of stress. Parental concerns and barriers to changing children's dietary and physical activity habits. Appetite 87: 137-142.

Park, S., L. Pan, B. Sherry and R. Li (2014). The association of sugar-sweetened beverage intake during infancy with sugar-sweetened beverage intake at 6 years of age. Pediatrics 134 Suppl 1: S5662 .

Pocock, M., D. Trivedi, W. Wills, F. Bunn and J. Magnusson (2009). Parental perceptions regarding healthy behaviours for preventing overweight and obesity in young children: a systematic review of qualitative studies. Obes Rev 11(338-353).

Rollins, B. Y., J. S. Savage, J. O. Fisher and L. L. Birch (2016). Alternatives to restrictive feeding practices to promote self-regulation in childhood: a developmental perspective. Pediatr Obes 11(5):326-32.

Rutter, J. and K. Stocker (2014). Childcare costs survey 2014, Family and Childcare Trust.

Shrewsbury, V. and J. Wardle (2008). Socioeconomic status and adiposity in childhood: a systematic review of cross-sectional studies 1990-2005. Obesity (Silver Spring) 16(2): 275-284.

Sleddens, E. F., S. M. Gerards, C. Thijs, N. K. de Vries and S. P. Kremers (2011). General parenting, childhood overweight and obesity-inducing behaviors: a review. Int J Pediatr Obes 6(2-2): e12-27.

Smith, P. K. (2005). The psychology of grandparenthood: An international perspective. London and New York Taylor \& Francis.

Sobal, J. and C. A. Bisogni (2009). Constructing food choice decisions. Ann Behav Med 38 Suppl 1: S37-46.

Styles, J. L., A. Meier, L. A. Sutherland and M. K. Campbell (2007). Parents' and caregivers' concerns about obesity in young children: a qualitative study. Fam Community Health 30(4): 279-295. 
Svensson, V., A. Ek, M. Forssen, K. Ekbom, Y. Cao, M. Ebrahim, E. Johansson, H. Nero, M. Hagstromer, M. Ekstedt, P. Nowicka and C. Marcus (2014). Infant growth is associated with parental education but not with parental adiposity - Early Stockholm Obesity Prevention Project. Acta Paediatr 103(4): 418-425.

U.S. Census Bureau. (2010). U.S. Census 2010. Retrieved 2010-11-18, 2010.

van der Horst, K., S. Kremers, I. Ferreira, A. Singh, A. Oenema and J. Brug (2007). Perceived parenting style and practices and the consumption of sugar-sweetened beverages by adolescents. Health Educ Res 22(2): 295-304.

Wijlaars, L. P., L. Johnson, C. H. van Jaarsveld and J. Wardle (2011). Socioeconomic status and weight gain in early infancy. Int J Obes (Lond) 35(7): 963-970.

Visser, S. S., I. Hutter and H. Haisma (2016). Building a framework for theory-based ethnographies for studying intergenerational family food practices. Appetite 97: 49-57.

World Health Organization (2000). Obesity: Preventing and managing the global epidemic of obesity. Report of a WHO consultation. WHO Technical Report Series. Geneva.

World Health Organisation Assembly Resolution. (2010). WHA63.14.

World Health Organization (2015). Guideline: Sugars intake for adults and children. Geneva. 